\title{
Graphene-Based Heterojunction between Two Topological Insulators
}

\author{
Oleksii Shevtsov, ${ }^{1}$ Pierre Carmier, ${ }^{1}$ Cyril Petitjean, ${ }^{1,2}$ Christoph Groth, ${ }^{1}$ David Carpentier, ${ }^{2}$ and Xavier Waintal ${ }^{1}$ \\ ${ }^{1}$ CEA-INAC/UJF Grenoble 1, SPSMS UMR-E 9001, Grenoble F-38054, France \\ ${ }^{2}$ CNRS-Laboratoire de physique, Ecole Normale supérieure de Lyon, France
}

(Received 24 January 2012; published 19 July 2012)

\begin{abstract}
Quantum Hall (QH) and quantum spin Hall (QSH) phases have very different edge states and, when going from one phase to the other, the direction of one edge state must be reversed. We study this phenomenon in graphene in the presence of a strong perpendicular magnetic field on top of a spin-orbit (SO)-induced QSH phase. We show that, below the SO gap, the QSH phase is virtually unaffected by the presence of the magnetic field. Above the $\mathrm{SO}$ gap, the $\mathrm{QH}$ phase is restored. An electrostatic gate placed on top of the system allows the creation of a QSH-QH junction which is characterized by the existence of a spin-polarized chiral state, propagating along the topological interface. We find that such a setup naturally provides an extremely sensitive spin-polarized current switch which could pave the way to novel spin-based electronic devices.
\end{abstract}

DOI: 10.1103/PhysRevX.2.031004 Subject Areas: Graphene, Mesoscopics, Spintronics, Topological Insulators

\section{INTRODUCTION}

Electronic properties of graphene and topological insulators have received considerable attention these last few years [1,2]. Graphene is a two-dimensional crystal whose electronic band structure is that of a gapless semiconductor, with conduction and valence bands touching each other at two inequivalent points, $\mathrm{K}$ and $K^{\prime}$, commonly referred to as valleys. The energy of charge carriers vanishes at these points and disperses linearly with momentum in their vicinity, forming a so-called Dirac cone. Low-energy excitations are massless Dirac fermions. These exotic quasiparticles carry a topological Berry phase which has been shown to give rise to many unusual transport phenomena such as the suppression of backscattering (also known as Klein tunnelling) $[3,4]$, weak antilocalization $[5,6]$, and a "relativistic" quantum Hall effect [7-9]. Some of these properties, however, are not robust to the presence of disorder-as soon as the latter is sufficiently short ranged to induce valley mixing-due to the existence in graphene of an even number of Dirac cones.

On the other hand, the recently discovered topological insulators are insulating in the bulk and characterized by the existence of robust gapless excitations at their surface [2,10-14]. In two dimensions, the topologically insulating phase possesses gapless states propagating along its edges. There are two famous examples of this phase: the quantum Hall $(\mathrm{QH})$ insulator, which can be obtained by applying a strong magnetic field perpendicular to the plane and is characterized by chiral spin-degenerate edge states [Fig. 1(b)], and the time-reversal-symmetric quantum spin Hall (QSH) insulator, which is induced by a strong

Published by the American Physical Society under the terms of the Creative Commons Attribution 3.0 License. Further distribution of this work must maintain attribution to the author(s) and the published article's title, journal citation, and DOI. spin-orbit (SO) interaction $[10,12,15]$ and is characterized by states with opposite spins propagating in opposite directions [Fig. 1(a)].

Whereas examples of three-dimensional topological insulators have been found to abound in nature, twodimensional systems exhibiting the QSH phase are so far limited to $\mathrm{HgTe} / \mathrm{CdTe}$ heterostructures, which only a few experimental groups in the world can synthesize. It was recently suggested [16] that graphene might come to the rescue, much like it did with two-dimensional electron gases, which were until a few years ago limited to epitaxially grown semiconducting heterostructures. Graphene's versatile fabrication methods and insensitivity to ambient temperature and chemical conditions have indeed raised important expectations for all sorts of applications in electronics [17]. Following the seminal observation by Kane and Mele that graphene with SO coupling should be a QSH insulator [10], Weeks et al. [16] numerically predicted the possibility of opening a substantial QSH gap in graphene by depositing on it heavy adatoms (such as $\mathrm{In}$ or $\mathrm{Tl}$ ), which can locally induce strong SO coupling in the system. In a previous paper [18], we studied in detail the transport in graphene randomly covered by diluted adatoms and showed that the QSH phase was insensitive to the inhomogeneity in the coverage $n_{\mathrm{ad}}$ and that, for all purposes, the system could be mapped to a homogeneous phase with a renormalized SO coupling strength $\lambda_{\mathrm{so}}^{\text {eff }}=\lambda_{\mathrm{so}} n_{\mathrm{ad}}$. We explore in this article the fate of the QSH phase when a strong perpendicular magnetic field is applied to graphene. Surprisingly, we show that the QSH phase is preserved for energies below the QSH gap, even for extremely strong magnetic fields or in the presence of disorder. As we will see, this can be traced back to the peculiar relativistic aspect of the $\mathrm{QH}$ effect in graphene (as well as to the negligible contribution of Zeeman splitting in graphene). The often-quoted simplified picture of SO coupling acting as an effective magnetic field with opposite signs for 

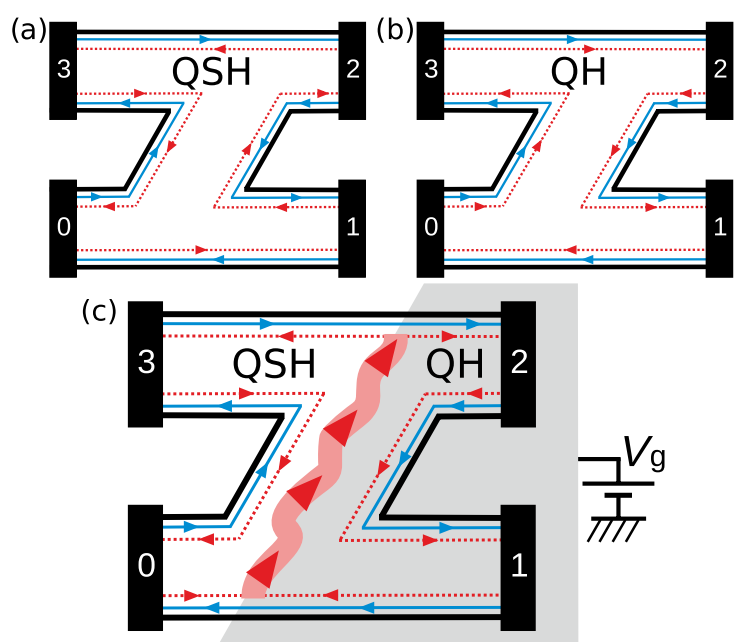

FIG. 1. Schematics for the edge states in a four-terminal Z-shape geometry when the system is in the (a) QSH phase and the (b) QH phase. In (a), opposite spins (thick blue lines and red dotted lines) propagate in opposite directions on a given edge, while in (b), they propagate in the same direction. (c) Topological heterojunction, with QSH edge states on the left and QH edge states on the right. This junction can be achieved experimentally by applying a top gate (shaded region) on the right half of the sample. While one of the spin species (thick blue line) can propagate through this junction, the other one (red dotted line) cannot, giving rise to a chiral state, localized at the interface between QH and QSH phases, which connects both edges.

opposite spins clearly breaks down in this situation. Next, we turn to the investigation of the transport properties of a junction between a QSH and a QH phase [Fig. 1(c)]. We show that this setup features a robust state, localized at the interface between the two topological insulators, analogous to the ambipolar "snake" states that arise in graphene quantum Hall $n-p$ junctions [19], and take advantage of this state by demonstrating how it can serve to realize a topologically protected spinpolarized charge-current switch.

The paper is organized as follows. In Sec. II, we recall the basic features of the QH and QSH phases and introduce the physical question at the root of this work. In Sec. III, we discuss the band structure of our system, and we show in Sec. IV how the use of topological Chern numbers can provide us with a clean and simple interpretation for the band structure. Numerical transport calculations are presented in Sec. V, in which we show that all regimes (below, above, and at the topological transition) possess characteristic signatures. The effects of geometry and disorder are discussed. Transport in the presence of a heterojunction between our two topologically insulating phases is analyzed in Sec. VI. There, we discuss similarities with other setups such as quantum Hall $n-p$ junctions, and comment on the possibility of realizing a spin-polarized current switch with huge on/off ratios. Our results are summarized in Sec. VII.

\section{HOW TO REVERSE THE DIRECTION OF PROPAGATION OF AN EDGE STATE}

The issue of how time-reversal symmetry breaking can affect the QSH phase has been addressed previously in the literature in different settings [20-23]. To the best of our knowledge, however, transport signatures of the competition between QH and QSH phases in graphene have not been considered yet, with the exception of the work by Abanin et al. [24], in which the QSH phase arose from a different mechanism (Zeeman splitting), which is extremely weak [25] and can therefore easily be destroyed by local fluctuations of the magnetic field. In contrast, we study the model introduced by Kane and Mele [10], to which we add the presence of a strong perpendicular magnetic field:

$$
H=v_{\mathrm{F}}\left(\hat{\Pi}_{x} \sigma_{x} \tau_{z}+\hat{\Pi}_{y} \sigma_{y}\right)+\Delta_{\mathrm{so}} \sigma_{z} \tau_{z} s_{z} .
$$

$\hat{\mathbf{I}}=\hat{\mathbf{p}}+e \mathbf{A}$ is the generalized momentum, which accounts for the presence of the magnetic vector potential $\mathbf{A}$ associated with a perpendicular magnetic field $\mathbf{B}=B \mathbf{z}$ $(\nabla \times \mathbf{A}=\mathbf{B}) ; \Delta_{\text {so }}$ is the SO-induced QSH gap; and $v_{\mathrm{F}}=$ $3 t a_{C-C} /(2 \hbar)$ is the Fermi velocity [expressed as a function of the microscopic lattice parameters $t$ (nearest-neighbor hopping amplitude) and $a_{C-C}$ (nearest-neighbor distance), which we choose in the following discussion as our working units of energy and length, respectively]. $\{\sigma, \tau, s\}$ are Pauli matrices in, respectively, sublattice, valley, and spin spaces.

The band structures of a graphene ribbon in the QSH and $\mathrm{QH}$ phases are quite different. In the $\mathrm{QH}$ phase, the perpendicular magnetic field gives rise to Landau levels $\epsilon_{n}= \pm\left(\hbar v_{\mathrm{F}} / l_{B}\right) \sqrt{2|n|}$, with $l_{B}=\sqrt{\hbar /(e B)}$, which become dispersive close to the edges of the graphene ribbon [Fig. 2(a)]. When the Fermi level is placed between two of these Landau levels, transport is characterized by spindegenerate edge states, as shown in Fig. 1(b), which propagate in a direction imposed by the sign of the magnetic field. In the QSH phase, the band structure consists of hyperbolic bands above the QSH gap and a pair of linearly dispersing bands below it [Fig. 2(b)]. These linear bands correspond to spin-polarized states, localized at the edges of the graphene ribbon on a characteristic length scale $\xi_{\text {so }}=\hbar v_{\mathrm{F}} / \Delta_{\text {so }}$. When the Fermi level is below the QSH gap, transport in the system can be described by counterpropagating edge states, as shown in Fig. 1(a). By comparing Figs. 1(a) and 1(b), one observes that one spin species-hereafter referred to as the "unhappy" spin (dotted red lines in Fig. 1) - has to reverse its direction of propagation when going from one phase to the other. This article is devoted to the study of how this reversal happens in two different setups: a homogeneous sample, 
where the quantum phase transition between QH and QSH phases is driven by electrostatic doping, and a heterojunction between the two phases.

\section{BAND STRUCTURE}

The first insight is given by the spectrum of Eq. (1) in the presence of graphene edges. The energy spectrum for a bulk system described by Eq. (1) reads $\epsilon_{n}= \pm \sqrt{\Delta_{B}^{2}|n|+\Delta_{\text {so }}^{2}}$, with $\Delta_{B}=\left(\hbar v_{\mathrm{F}} / l_{B}\right) \sqrt{2}$. The lowest level $n=0$ stands out from the others, as each branch $( \pm)$ can be shown to host only one of the two spin species [22] [Fig. 2(c)]. Whereas $n>0$ levels will all disperse in the same direction when confinement is taken into account, the fate of the lowest level is more subtle. To be more quantitative, we make use of a tight-binding model on the graphene hexagonal lattice, which in the presence of both Kane-Mele SO coupling and perpendicular magnetic field can be written as

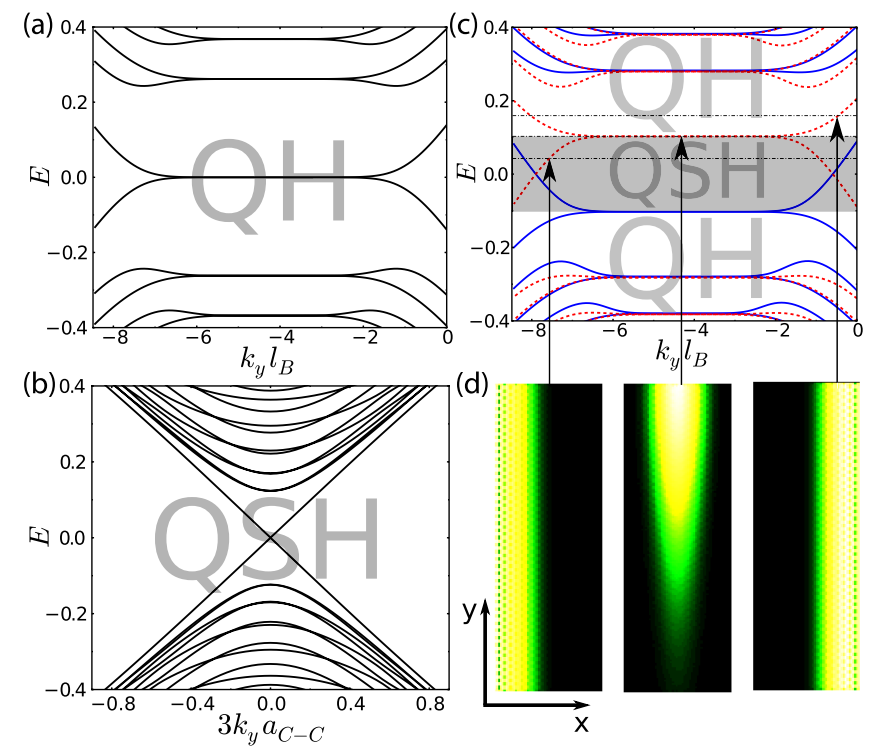

FIG. 2. Band structure of a (semimetallic armchair) graphene ribbon in the (a) QH and (b) QSH phase. When both magneticfield coupling and SO coupling are present (c), the resulting band structure leads to a QSH phase for $\left|E_{\mathrm{F}}\right|<\Delta_{\text {so }}$ (shaded region) and a $\mathrm{QH}$ phase for $\left|E_{\mathrm{F}}\right|>\Delta_{\mathrm{so}}$. Compared to the pure $\mathrm{QH}$ and QSH cases, the spin degeneracy is lifted (blue thick lines and red dotted lines), seen as particularly prominent in the lowest band which consists of spin-polarized branches at $E= \pm \Delta_{\text {so }}$. As the Fermi energy crosses the SO gap, the localization of the unhappy spin (red dotted lines) shifts from one edge to the other, while it is fully localized in the bulk when $E_{\mathrm{F}}=\Delta_{\mathrm{so}}$. This is illustrated in the corresponding current-density plots (d). On the other hand, the happy spin (thick blue) gets increasingly localized on the same edge as the Fermi energy crosses the transition region (not shown). The parameters used are $\lambda_{\text {so }}=0.02, l_{B} \simeq 8$, and a ribbon width $W=40 \sqrt{3}$.

$$
H=-t \sum_{\langle i, j\rangle, \alpha} e^{i \phi_{i j}} c_{i, \alpha}^{\dagger} c_{j, \alpha}+i \lambda_{\mathrm{so}} \sum_{\langle\langle i, j\rangle\rangle, \alpha, \beta} \nu_{i j} e^{i \phi_{i j}} c_{i, \alpha}^{\dagger} s_{\alpha \beta}^{z} c_{j, \beta}
$$

Indices $(i, j)$ label lattice sites, $(\alpha, \beta)$ label spin indices, while symbols \langle\rangle and $\langle\langle\rangle\rangle$ respectively refer to nearestneighbor coupling (with hopping amplitude $t$ ) and nextnearest-neighbor coupling (with SO-induced hopping amplitude $\left.\lambda_{\mathrm{so}}=\Delta_{\mathrm{so}} /(3 \sqrt{3})[10]\right)$. The Peierls phase $\phi_{i j}=$ $(e / \hbar) \int_{\mathbf{r}_{j}}^{\mathbf{r}_{i}} \mathbf{A} \cdot d \mathbf{r}$ takes into account the contribution from the magnetic flux threading the lattice, and $\nu_{i j}= \pm 1$ depending on whether sites are coupled clockwise or counterclockwise. Note that, in order for the system to remain gauge invariant, Peierls substitution has to be performed on all hopping matrix elements: nearest-neighbor and (SO) next-nearest-neighbor. To compute transport properties numerically, we make use of the software KNIT, which is based on an advanced recursive Green's function technique [28], and we work in the linear response regime. The numerical calculations are done with semimetallic armchair boundary conditions, but our results are qualitatively unaffected by this choice. An important technical point is that the magnetic field should be present in the entire sample, including in the leads, in order to avoid spurious reflection at the lead-sample interface. The multiterminal Peierls-substitution prescription that allows us to do that is described in the Appendix.

The full tight-binding band structure of a semimetallic armchair graphene ribbon described by the Hamiltonian in Eq. (2) is shown in Fig. 2(c). It can be summarized in very simple terms: For Fermi energies inside the SO gap $\left|E_{\mathrm{F}}\right|<\Delta_{\text {so }}$ (shaded region), the system is in the QSH phase, with opposite spin channels on a given edge propagating in opposite directions, while for energies $\left|E_{\mathrm{F}}\right|>\Delta_{\text {so }}$, the system is in the QH phase, with opposite spin channels on a given edge propagating in the same direction. Hence, for a given value of $\Delta_{\text {so }}$, the transition between the two phases is governed solely by the Fermi energy and does not depend at all on the value of the magnetic field (once again neglecting Zeeman splitting, which is very small in graphene). This quite remarkable result is a direct consequence of the existence in graphene of a $B$-independent zero-energy Landau level: As soon as $\Delta_{\text {so }} \neq 0$, the spin degeneracy of the zero-energy Landau level is lifted, as opposed to all other Landau levels, which remain spin degenerate [22]. This lifting leads to a QSH phase in the corresponding SO gap, as can be understood with the help of topological invariants.

\section{TOPOLOGICAL ORDER}

In this section, we discuss the topological order of the phases obtained by varying the chemical potential in the energy spectrum of Fig. 2(c). In particular, we relate the unique transition between the QSH topological order 
and the $\mathrm{QH}$ topological order in the presence of $\mathrm{SO}$ coupling to the specificities of the $\mathrm{QH}$ physics of Dirac fermions in graphene. Let us start by recalling the standard topological-number characterization of Landau levels when $\Delta_{\text {so }}=0$. Each Landau level $n$ and its associated eigenfunctions over the first Brillouin zone are characterized by a topological invariant, the so-called Chern number [29]. This topological number takes a value $\mathcal{C}_{\tau, s}^{(n)}=+1$ for each Landau level, independent of the Landau $n$, valley $\tau$, or spin $s$ indices. For each value of the Fermi energy, we can characterize the corresponding phase by a topological number $\mathcal{C}=\sum_{\tau, s} \sum_{\epsilon_{n}<E_{\mathrm{F}}} \mathcal{C}_{\tau, s}^{(n)}$ obtained by summing the Chern numbers of all filled energy bands [29]. For graphene and any Dirac system, however, this procedure would yield an ill-defined topological number $\mathcal{C}$ due to the presence of an infinite number of filled Landau levels below $E_{\mathrm{F}}$. As shown recently through the use of the noncommutative Berry's connection [30], the correct topological number for a single Dirac cone takes a value $\mathcal{C}_{\tau, s}=-1 / 2$ for $\epsilon_{-1}<E_{\mathrm{F}}<\epsilon_{0}$, and $\mathcal{C}_{\tau, s}=+1 / 2$ for $\epsilon_{0}<E_{\mathrm{F}}<\epsilon_{1}$, where $\epsilon_{n}$ are the usual Landau levels and $\mathcal{C}_{\tau, s}=\sum_{\epsilon_{n}<E_{\mathrm{F}}} \mathcal{C}_{\tau, s}^{(n)}$. In the discussion below, we will make use of the more convenient topological Chern number per spin species, $\mathcal{C}_{s}=\sum_{\tau} \mathcal{C}_{\tau, s}$, which takes values twofold larger.

Let us now turn to the energy spectrum of Fig. 2(c), where $\Delta_{\text {so }} \neq 0$. The presence of the SO coupling does not modify any of the Chern numbers per Landau level, but it lifts the spin degeneracy of the $n=0$ Landau level into the two levels at $E= \pm \Delta_{\mathrm{so}}$. As the $z$ component of spin is conserved, the topological Chern numbers per spin species introduced above turn out to be useful quantities for characterizing the topological order in this new spectrum. They read

$$
\begin{array}{lll}
\mathcal{C}_{\uparrow}=-1, & \mathcal{C}_{\downarrow}=-1 & \text { for } \epsilon_{-1}<E_{\mathrm{F}}<-\Delta_{\text {so }}, \\
\mathcal{C}_{\uparrow}=+1, & \mathcal{C}_{\downarrow}=-1 & \text { for }-\Delta_{\text {so }}<E_{\mathrm{F}}<\Delta_{\text {so }}, \\
\mathcal{C}_{\uparrow}=+1, & \mathcal{C}_{\downarrow}=+1 & \text { for } \Delta_{\text {so }}<E_{\mathrm{F}}<\epsilon_{1}, \\
\mathcal{C}_{\uparrow}=+3, & \mathcal{C}_{\downarrow}=+3 & \text { for } \epsilon_{1}<E_{\mathrm{F}}<\epsilon_{2},
\end{array}
$$

this time with $\epsilon_{n}$ the modified Landau levels introduced at the beginning of Sec. III. The difference between $\mathcal{C}_{\uparrow}$ and $\mathcal{C}_{\downarrow}$ for $\left|E_{\mathrm{F}}\right|<\Delta_{\text {so }}$ signals the appearance of a $\mathbb{Z}_{2}$ topological order characteristic of the QSH phase. Indeed, when the $z$ component of spin is conserved, the $\mathbb{Z}_{2}$ topological index characterizing the QSH phase is defined as $\nu=$ $\left(\mathcal{C}_{\uparrow}-\mathcal{C}_{\downarrow}\right) / 2(\bmod 2)[2,31]$. As all Landau levels $n \neq 0$ are still spin degenerate, we have $\mathcal{C}_{\uparrow}=\mathcal{C}_{\downarrow}$ and thus $\nu=0$ for all Fermi energies $\left|E_{\mathrm{F}}\right|>\Delta_{\mathrm{so}}$. For these values of $E_{\mathrm{F}}$, the system lies in a $\mathrm{QH}$ phase characterized by the usual topological Chern number $\mathcal{C}=\mathcal{C}_{\uparrow}+\mathcal{C}_{\downarrow}$. However, for $\left|E_{\mathrm{F}}\right|<\Delta_{\text {so }}$, Eq. (3) leads to a nontrivial $\mathbb{Z}_{2}$ index $\nu=1$, while the total Chern number simultaneously vanishes $\mathcal{C}=\mathcal{C}_{\uparrow}+\mathcal{C}_{\downarrow}=0$. The system then lies in a different topologically insulating phase: the QSH insulator. This shows that as the Fermi energy crosses the values $\pm \Delta_{\mathrm{so}}$, the system undergoes a quantum phase transition between two topological insulators: a QH phase and a QSH phase:

$$
\begin{array}{ll}
\nu=0, & \mathcal{C}=-2 \text { for } \epsilon_{-1}<E_{\mathrm{F}}<-\Delta_{\text {so }}: \mathrm{QH}, \\
\nu=1, & \mathcal{C}=0 \text { for }-\Delta_{\text {so }}<E_{\mathrm{F}}<\Delta_{\text {so }}: \mathrm{QSH}, \\
\nu=0, & \mathcal{C}=2 \text { for } \Delta_{\text {so }}<E_{\mathrm{F}}<\epsilon_{1}: \mathrm{QH} \\
\nu=0, & \mathcal{C}=6 \text { for } \epsilon_{1}<E_{\mathrm{F}}<\epsilon_{2}: \mathrm{QH} .
\end{array}
$$

This transition appears crucially tied to the Dirac physics of graphene and the presence of the $n=0$ Landau level: In the present case, we do not need a SO coupling to overcome an energy gap in order to drive this transition, as would be the case for nonrelativistic fermions with Landau gap $\hbar \omega_{c}$ or if graphene had a trivial mass gap $m \sigma_{z}$. The spin-degeneracy lifting of the $n=0$ level is all that is required here. Let us note finally that, while this argument formally uses the conservation of the $S_{z}$ spin component, the robustness of topological numbers proves it to remain valid if non- $S_{z}$-conserving terms are included in the Hamiltonian, provided two branches of the $n=0$ Landau level with opposite spins remain nondegenerate.

\section{TRANSPORT SIGNATURES}

\section{A. Ballistic regime}

Let us now study how this topological phase transition appears in transport. We will study the multiterminal (dimensionless) differential conductance $T_{a b}$, which expresses how much current $d I_{a}$ is collected in lead $a$ when the voltage in lead $b$ is raised by $d V_{b}$ :

$$
\frac{d I_{a}}{d V_{b}}=\frac{e^{2}}{h} T_{a b}
$$

Additionally, in order to observe the edge states directly, we also study (in color plots) the differential local current density $\operatorname{di}(\vec{r}) / d V_{a}$, which allows us to clearly observe the edge states inside the sample. In Fig. 2(d), we show local current-density plots which illustrate how the behavior of the unhappy spin changes as a function of the Fermi energy: It goes from propagating along one edge when $E_{\mathrm{F}}<\Delta_{\text {so }}$ (left panel) to propagating along the other as $E_{\mathrm{F}}>\Delta_{\text {so }}$ (right panel), while it gets localized in the bulk at the critical point $E_{\mathrm{F}}=\Delta_{\text {so }}$ (middle panel). We find that the energy window where this localization is observed is extremely narrow and decays exponentially with the width of the sample. Note that this scenario is completely different from what one would expect starting from the naïve toy model of SO coupling acting as a spin-dependent magnetic field $B_{\mathrm{so}} \mathbf{z} s_{z}$. In this case, for a critical value of the real magnetic field $B=B_{\mathrm{so}}$, the unhappy spin would feel no magnetic field at all and be fully delocalized. On the contrary, we observe that the QSH phase is virtually independent from the real magnetic field and that the unhappy 
spin actually gets localized when $E_{\mathrm{F}}=\Delta_{\mathrm{so}}$, illustrating the limitations of the toy model in this situation.

In the vicinity of the transition, the unhappy spin keeps propagating along a given edge but its classical cyclotron orbit center $x_{\mathrm{c}}=-k_{y} l_{B}^{2}$ (with $k_{y}$ the longitudinal wave vector component) is shifted inwards as the Fermi energy increases [see Fig. 2(c)]. On the other hand, the "happy" spin gets increasingly localized on the same edge when the Fermi energy increases, which is qualitatively equivalent to the usual QH case. One can indeed show that the notion of a classical cyclotron orbit center remains well defined here, despite the presence of SO coupling and that the corresponding eigenstates are very similar to those found in the pure $\mathrm{QH}$ regime [22].

Before presenting the rest of our numerical data on transport in the vicinity of the QSH-QH transition, we should say a few words about the shape of the spectrum corresponding to the nonzero Landau levels. While the branches corresponding to the happy spin seem basically unaffected by the SO coupling, the two branches of the unhappy spin display very different behaviors [Fig. 2(c)]. In particular, one of them is significantly bent by the SO coupling, such that counterpropagating states along the same edge appear in a finite window of energy. This leads to the possibility of backscattering and therefore destroys the robustness of the $\mathrm{QH}$ phase in this energy window, the size of which can nevertheless be significantly reduced by increasing the width of the graphene ribbon. Note that, at negative energies, $E_{\mathrm{F}}<-\Delta_{\mathrm{so}}$, happy and unhappy spin species exchange their roles [see Fig. 2(c)].

We proceed to investigate further how the transition between QSH and QH phases appears in transport, and consider the four-terminal $\Psi$-shape geometry depicted in the inset of Fig. 3(a). In the core of Fig. 3(a), we plot as a function of the Fermi energy the current collected in leads 1,2 , and 3 when injected from lead 0 . Nothing unexpected happens away from the transition: The transmission coefficients feature characteristic signatures of current-carrying edge states [left and right panels of Fig. 2(d)]. Around the critical value $E_{\mathrm{F}}=\Delta_{\text {so }}$, however, we observe in Fig. 3(a) that the unhappy spin is fully transmitted in lead 2. This can be understood as follows. As the transition point is approached, the classical cyclotron orbit center is shifted (inwards) away from the edge (as discussed above) by a distance which can reach $2 R_{\mathrm{c}}$, where $R_{\mathrm{c}}=l_{B}^{2} / \xi_{\mathrm{so}}$ is the classical cyclotron radius of the $n=0$ level. When $2 R_{\mathrm{c}}>W_{1} / 2$, where $W_{1}$ is the width of lead 1 , then, somewhere in the vicinity of the transition, the incoming state cannot penetrate lead 1 , hence leading to perfect direct transmission to lead 2, as observed in Fig. 3(a). This feature could in principle be checked experimentally by simply varying the strength of the magnetic field close to the transition: For wider systems or at larger magnetic field, the width of the peak of direct transmission from 0 to 2 shrinks and eventually disappears.
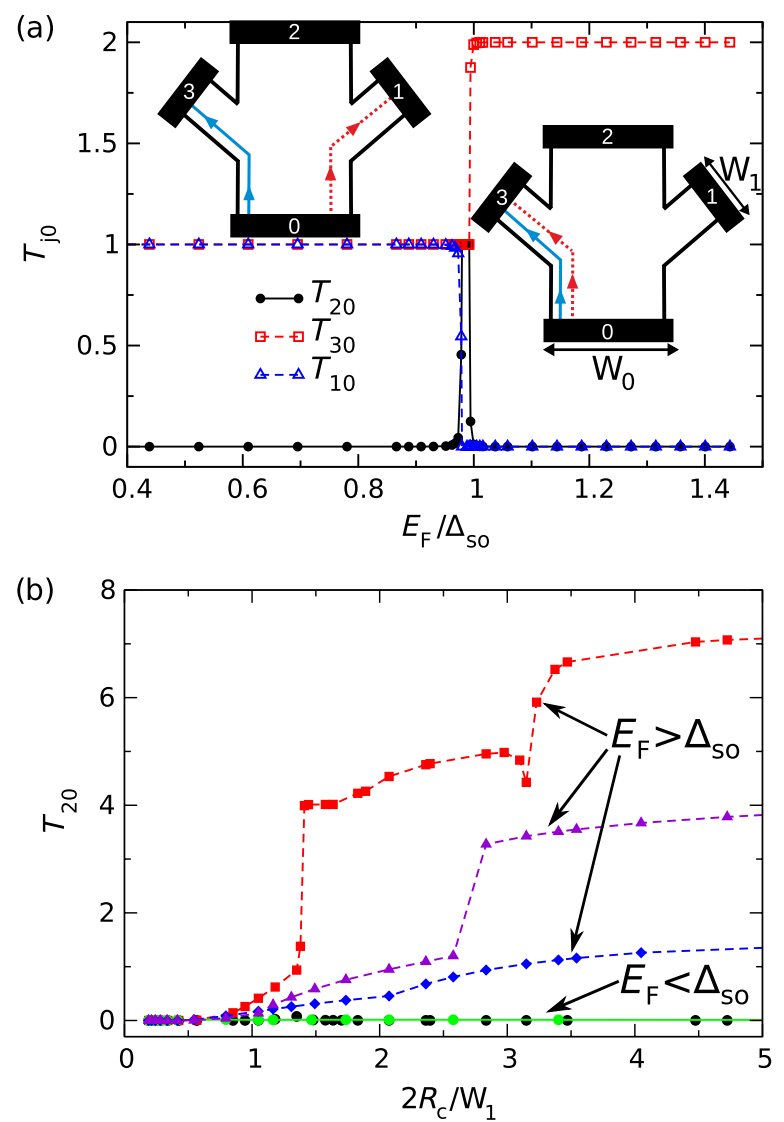

FIG. 3. QSH-QH transition in a four-terminal $\Psi$-shape geometry. (a) Dimensionless differential conductance from lead 0 , where the current is injected, to outgoing leads 1,2 , and 3 in the geometry depicted in the inset, as a function of the Fermi energy $\left(\lambda_{\mathrm{so}}=0.02, l_{B} \simeq 8\right.$, and lead widths $W_{0}=64 \sqrt{3}$, $W_{1}=22 \sqrt{3}$ ). Below the SO gap, current is carried by QSH edge states, which propagate on different edges (left inset), while above the SO gap it is carried by $\mathrm{QH}$ edge states, which propagate on the same edge (right inset). (b) Dimensionless differential conductance from lead 0 to lead 2 as a function of the classical cyclotron radius $R_{\mathrm{c}}$ [same values of $\lambda_{\mathrm{so}}, W_{0}$, and $W_{1}$ as in (a)]. Circular symbols correspond to data with $E_{\mathrm{F}}<\Delta_{\text {so }}$, while other symbols (dashed lines) correspond to data with $E_{\mathrm{F}}>\Delta_{\mathrm{so}}$ : respectively, $E_{F} / \Delta_{\mathrm{so}}=1.06$ (blue diamonds); $E_{\mathrm{F}} / \Delta_{\text {so }}=1.25$ (violet triangles); and $E_{\mathrm{F}} / \Delta_{\text {so }}=1.44$ (red squares). While this direct transmission is vanishingly small and independent of the magnetic field below the SO gap, it increases with both $R_{\mathrm{c}}$ and $E_{\mathrm{F}}$ above the $\mathrm{SO}$ gap. The latter situation arises because the $\mathrm{QH}$ phase is destroyed as $2 R_{\mathrm{c}} \gtrsim W_{1}$, and because the number of transmitting channels increases with $l_{B}$ and $E_{\mathrm{F}}$.

A clear way to distinguish between QH and QSH phases in a transport measurement is to plot the "direct" dimensionless differential conductance from lead 0 to lead 2 as a function of the classical cyclotron radius $R_{\mathrm{c}} \propto B^{-1}$. This is illustrated in Fig. 3(b): Below the QSH gap, the direct transmission is zero for any value of the magnetic field; on the other hand, above the QSH gap, transmission can 
become nonzero as the magnetic field weakens, due to the breakdown of the QH effect when $R_{\mathrm{c}}$ becomes larger than the width of the system. It thus interpolates between zero (in the $\mathrm{QH}$ regime) and a finite value which depends on the number of occupied bands at the Fermi level in the ribbon when $B=0$. Of course, in the latter limit, the topological protection is lost and the value of the transmission will strongly depend on the disorder configuration.

\section{B. Effect of disorder}

In this section, we study the robustness of the results described in the ballistic regime with respect to the presence of various types of disorder. In the absence of a magnetic field, the QSH phase is known to be very robust with respect to the presence of scalar disorder (i.e., disorder that breaks neither time-reversal symmetry nor spin-rotational symmetry), as introducing backscattering between edge states involves tunneling through the gapped bulk region. On the other hand, disorder that breaks both time-reversal symmetry and spin-rotational symmetry (such as magnetic impurities) leads to scattering between the two counterpropagating edge states of the same edge, which leads to intraedge backscattering. If this intraedge backscattering becomes strong (or the edges are very long), edge states may eventually get localized, such that no edge transport occurs anymore. In this paper, as we explicitly break time-reversal symmetry with a magnetic field, disorder that breaks only spin-rotational symmetry (such as Rashba-like SO terms arising from adatoms) effectively behaves as magnetic impurities and could potentially lead to the same breakdown of the QSH phase.

In order to study these effects quantitatively, we add the general perturbation

$$
H_{\mathrm{dis}}=\sum_{i, \alpha, \beta} c_{i, \alpha}^{\dagger}\left(\sum_{\mu=0, x, y, z} V_{i, \mu} s_{\alpha \beta}^{\mu}\right) c_{i, \beta}
$$

to our tight-binding Hamiltonian Eq. (2). The onsite potentials $V_{i, \mu}$ are independent random variables uniformly distributed inside a given interval on each site of the system (Anderson disorder). We study three different sorts of disorder with different symmetries: $V_{i, 0} \in\left[-V_{\mathrm{s}} / 2, V_{\mathrm{s}} / 2\right]$ takes into account scalar (spin-independent) disorder; $V_{i, z} \in$ $\left[-V_{\mathrm{z}} / 2, V_{\mathrm{z}} / 2\right]$ represents Zeeman-like $\left(S_{\mathrm{z}}\right.$-conserving) impurities, and $\left\{V_{i, x}, V_{i, y}\right\} \in\left[-V_{\mathrm{m}} / 2, V_{\mathrm{m}} / 2\right]$ captures the influence of $S_{\mathrm{z}}$-nonconserving impurities.

The results are presented in Fig. 4 for the four-terminal $\Psi$-shape geometry [depicted in the inset of Fig. 3(a)]. Note that, in order to obtain a significant effect of disorder, we used extremely (unrealistic) high values of disorder, much higher than the SO gap itself. A first general qualitative conclusion is that the ballistic results presented above are extremely robust with respect to all kinds of disorder. More precisely, we find, as expected, that extremely large values of $V_{\mathrm{s}}$ or $V_{\mathrm{z}}$ are needed to significantly affect the transport coefficients. "Magnetic" impurities $\left(V_{\mathrm{m}}\right)$, which can

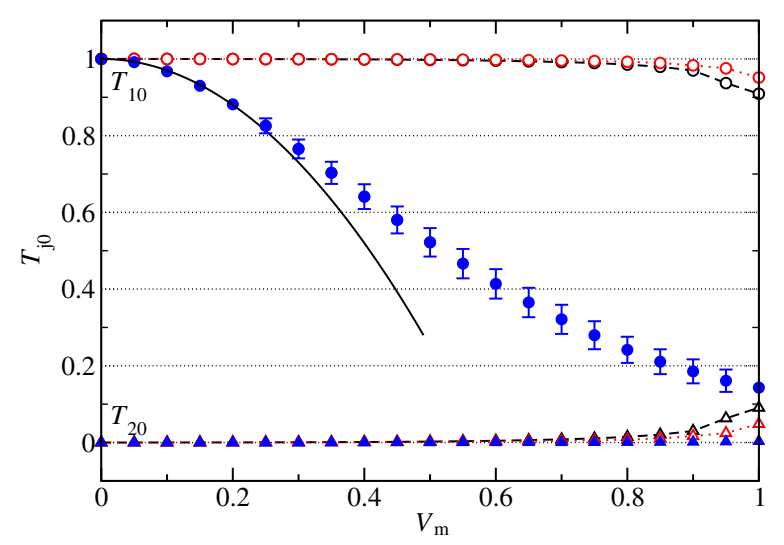

FIG. 4. Effect of very strong disorder on the QSH phase in the four-terminal $\Psi$-shape geometry. We plot the dimensionless differential conductance from lead 0 to leads 1 and 2 as a function of disorder strength for $\lambda_{\text {so }}=0.02, l_{B} \simeq 8, E_{\mathrm{F}}=$ 0.05, and otherwise the same system as in Fig. 3. Circular symbols stand for $T_{10}$ and triangular symbols for $T_{20}$. The behavior for $T_{30}$ (not shown) is the same as for $T_{10}$. Dashed black lines correspond to purely scalar disorder $\left(V_{\mathrm{s}}\right)$, dotted (red) lines correspond to purely Zeeman-like disorder $\left(V_{z}\right)$, and filled blue symbols correspond to purely $S_{\mathrm{z}}$ nonconserving disorder $\left(V_{\mathrm{m}}\right)$. The latter is clearly the dominant effect on $T_{10}$, although unrealistically large values are required to quantitatively affect the edge-state transmission. (See main text for a mean-free-path estimate.) Each data point has been averaged over 48 disorder configurations. Unless shown, error bars are smaller than symbol sizes. Full black line: fit $y=1-3.0 x^{2}$.

induce intraedge backscattering, do affect the QSH phase at weaker values. We note, however, that the system remains topological (i.e., it does not become a simple ordinary insulator), as transport is still dominated by edge contributions. For instance, at $V_{\mathrm{m}}=0.5$, the direct transmission probability $T_{20}$ (from lead 0 to lead 2 ) remains many orders of magnitude smaller than $T_{10}$ (from lead 0 to lead 1). The topological nature of the phase is encoded in the $\mathbb{Z}_{2}$ topological invariant, which cannot change unless the bulk gap is closed by the perturbations we consider. We checked (not shown) that the above results are essentially unaffected upon changing the SO gap by a factor of 2, putting all three sorts of disorder simultaneously, or changing the Fermi energy (inside the SO gap).

To rule out any concerns raised by the intraedge scattering due to $V_{\mathrm{m}}$, the corresponding intraedge mean free path $l_{e}$ (or equivalently the localization length, as both are roughly equal for one-dimensional states) can be estimated using Fermi's golden rule: $l_{\mathrm{e}}=v_{\mathrm{d}} \tau_{\mathrm{e}}$, where $v_{\mathrm{d}}=$ $\hbar^{-1}(d E / d k)$ is the drift velocity of the edge states (extracted, for instance, from Fig. 2), and $\hbar / \tau_{\mathrm{e}} \propto$ $\left(V_{\mathrm{m}}\right)^{2}|d k / d E|$. Alternatively, one may extract it directly from the numerical calculations of Fig. 4, since $T_{10} \approx 1-\left(L / l_{\mathrm{e}}\right)$ (with $L$ the length along the edge, or roughly $5 \mathrm{~nm}$, in this instance). Fitting the small $V_{\mathrm{m}}$ regime with $T_{10} \approx 1-3.0 V_{\mathrm{m}}^{2}$, we obtain (in $\mathrm{nm}$ ) 


$$
l_{\mathrm{e}} \approx\left(V_{\mathrm{m}} / t\right)^{-2},
$$

where we have explicitly restored the hopping amplitude $t$ (our energy unit) in order to get numbers. The ballistic results are essentially unaffected for systems smaller than $l_{\mathrm{e}}$, while the edge states become localized for larger systems. In the context of a QSH phase induced by adatoms (using, for instance, the indium atoms proposed by Weeks et al. [16]), a possible source of $V_{\mathrm{m}}$-like disorder comes from the SO coupling induced by the adatoms themselves. Typical values for $V_{\mathrm{m}}$ are smaller than $1 \mathrm{meV}$, which translates into extremely large intraedge mean free paths $l_{\mathrm{e}}>1 \mathrm{~mm}$. Hence, we estimate that this perturbation should be largely irrelevant in realistically sized samples.

To summarize, all of our results are essentially unaffected by the presence of disorder, except when the Fermi energy lies in the vicinity of the QSH-QH transition, in which case strong disorder can give rise to a random network of QH and QSH regions through which a percolating cluster connecting opposite edges can therefore lead to backscattering [32].

\section{TOPOLOGICAL HETEROJUNCTION}

We take advantage of the above-described topological quantum phase transition as a function of the Fermi energy to propose a setup that allows for a direct junction between two different topological phases in the same sample. Let us consider the case in which an additional electrostatic gate enables one to split the system in two parts: one in which the Fermi level is in the QSH phase, and the other in which the Fermi level is in the QH phase [Fig. 1(c)]. This constitutes a QSH-QH junction, which shares some similarities with quantum Hall $n-p$ junctions previously fabricated in graphene $[34,35]$. Indeed, the incoming unhappy spin at the junction has no choice but to propagate along the interface in order to reach the only other available channels that lie on the opposite edge. This is reminiscent of the situation both spin channels must face in the $\mathrm{QH}$ regime when they must cross a $n-p$ junction, since their direction of propagation on a given edge is reversed for negative energies. Various theoretical models have been proposed in the latter setup [36-39], but they all fail to explain the experimental observations $[34,35]$, probably due to some dephasing mechanism taking place in the vicinity of the Dirac point which is obscured by chargedensity fluctuations (so-called electron-hole puddles). The system we consider could therefore provide a new perspective for solving this puzzle, as the QSH-QH transition takes place at a value of energy which can be far away from the Dirac point [40] for realistic values of SO-induced QSH gap.

More generally, our proposal offers the possibility of studying the nature of the state that propagates at the interface between QSH and QH phases, which are characterized by different topological invariants [11,29]. What we usually refer to as QSH (or QH) edge states are states propagating between QSH (or QH) insulators and a trivial insulator (the vacuum, typically). As the QSH insulator is characterized by a $\mathbb{Z}_{2}$ number, there is only one QSH topological phase: Junctions between QSH phases with different Fermi energies (including $n-p$ junctions) have no effect on transport, as the spin-polarized states can propagate through these junctions. On the other hand, the QH topological invariant is a $\mathbb{Z}$ number, which counts the number of edge channels, and the notion of $\mathrm{QH}$ junctions therefore makes sense. In this case, one expects the existence of chiral propagating states, localized at the interface corresponding to the Landau level crossing. For QH $n-n^{\prime}$ junctions, these states are "bubbling" states [39], which simply follow the drifting Hall motion of charge carriers subjected to crossed electric and magnetic fields. For QH $n-p$ junctions, these states are ambipolar snake states [19], which can be seen as classical skipping orbits of mixed electron-"hole" character. The characteristics of the state propagating at the interface between $\mathrm{QSH}$ and $\mathrm{QH}$ insulators, on the other hand, are still unclear as far as we know.

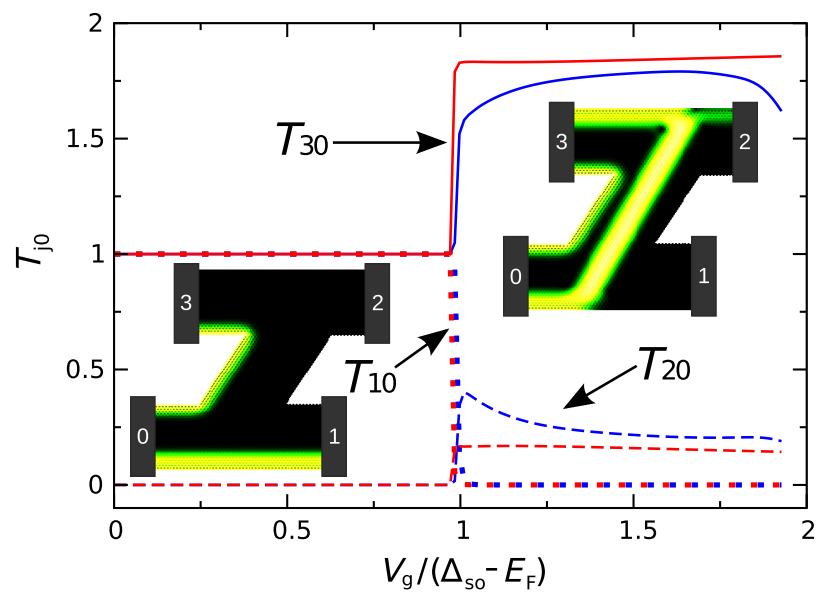

FIG. 5. Heterostructure in a four-terminal Z-shape sample as depicted in Fig. 1(c). Transmission probabilities from lead 0, where the current is injected, to outgoing leads 1 (dotted lines), 2 (dashed lines), and 3 (solid lines) as a function of the top gate voltage $V_{\mathrm{g}}$. When $V_{\mathrm{g}}<\left(\Delta_{\mathrm{so}}-E_{\mathrm{F}}\right)$, such that left and right regions are in the QSH phase, current is perfectly transmitted by the QSH edge states, as shown in the current-density plot in the left inset. When $V_{\mathrm{g}}$ is high enough $\left[V_{\mathrm{g}}>\left(\Delta_{\text {so }}-E_{\mathrm{F}}\right)\right]$ that the right part of the sample enters the $\mathrm{QH}$ phase, a QSH-QH junction is created, characterized by a chiral state propagating along the interface. This is illustrated in the current-density plot shown in the right inset. Once it has reached the opposite edge, this chiral state is partially transmitted in lead 2 and partially transmitted in lead 3, with proportions shown in the main plot. The light red curves correspond to an abrupt voltage change across the junction region while the dark blue curves correspond to a smooth transition. Parameters are $E_{\mathrm{F}} / \Delta_{\mathrm{so}}=0.58$ (in the left half of the sample), $\lambda_{\text {so }}=0.02, l_{B} \simeq 8$, and widths $W_{0}=W_{\mathrm{c}}=40 \sqrt{3}$ for the leads and central region. 
Our proposal is as follows. Consider the Z-shape geometry shown in Fig. 1(c). It is split into two regions: the left half and the right half. A top gate is applied on the right half in order to tune the Fermi level in both regions independently. In Fig. 5, we plot, for a given value of the Fermi energy in the left half inside the QSH gap, the differential conductance $T_{i 0}$ from lead 0 to leads 1,2 , and 3 as a function of the gate voltage (which determines the value of the Fermi energy in the right half). While the Fermi energy in the right half remains below the value of $\Delta_{\text {so }}$, transport characteristics remain unaffected by the gate (left inset of Fig. 5). However, as soon as the Fermi energy in the right half crosses the QSH gap, it gives rise to a QSH-QH junction characterized by a topological state at its interface (right inset of Fig. 5). This chiral state propagates along the interface until it reaches the opposite edge, and then it gets partially transmitted in lead 2, and partially transmitted in lead 3, with proportions which depend on the microscopic details of the model. (Those details include Fermi energies, the length of the interface, the smoothness of the potential step, the amount of disorder, the possible valley-space polarization of the edge states, and so on, the study of which is left to subsequent work.) This system constitutes a very efficient spin-polarized, charge-current switching mechanism, as the current in lead 1 (respectively, 2) can be reversibly switched from one (respectively, zero) to zero (respectively, nonzero) while simultaneously being spin polarized (see Fig. 5). Additionally, this switching can be activated by simply tuning the voltage in the top gate over a very small energy range (whose lower bound will depend on the magnitude of the disorder in the vicinity of the transition), and should yield extremely sharp transitions with values of on/off current topologically protected from the presence of disorder [41].

\section{CONCLUSION}

In summary, we have shown that the transition between QSH and QH phases in graphene is independent of the value of the magnetic field (neglecting the weak effect of Zeeman splitting) and can be crossed simply by tuning the value of the Fermi energy across the SO-induced QSH gap. In a heterojunction, one of the spin species gives rise to a chiral state propagating along the interface between QSH and $\mathrm{QH}$ phases. The nature of this special state is a fascinating issue which could bring new light concerning the unresolved mystery of conductance plateaus observed in quantum Hall $n-p$ junctions [34,35]. We have also shown that the tunable transition between the QSH and QH topological phases could serve as a spin-polarized charge-current switch with potentially extremely high, topologically protected, on/off ratios. An interesting future direction of research could be to investigate whether this tunable topological phase transition can arise in bilayer graphene [42], which also possesses zero-energy Landau levels [43] and has very recently been shown to host a $\mathbb{Z}_{2}$ topologically insulating phase [44] for strong enough Rashba SO coupling.

\section{ACKNOWLEDGMENTS}

This work was supported by STREP ConceptGraphene, EC Contract No. ERC MesoQMC, and ANR Grant No. 2010-IsoTop.

\section{APPENDIX: MAGNETIC FIELD IN MULTITERMINAL CALCULATIONS}

In this appendix, we explain how to compute the Peierls phase $\phi_{i j}$ between two atoms $i$ and $j$ in multiterminal systems [45]. The phase is given by

$$
\phi_{i j}=\int_{\mathbf{r}_{j}}^{\mathbf{r}_{i}} \mathbf{A} \cdot d \mathbf{r},
$$

where $\mathbf{r}_{i}=\left(x_{i}, y_{i}\right)$ is the spatial position of site $i$. A common choice for $\mathbf{A}$ is the Landau gauge (Lg),

$$
\mathbf{A}_{\mathrm{Lg}}(x, y)=-B y\left(\begin{array}{l}
1 \\
0
\end{array}\right),
$$

which leads to

$$
\phi_{i j}^{\mathrm{Lg}}=-B\left(x_{i}-x_{j}\right) \frac{y_{i}+y_{j}}{2}
$$

(using linear paths between atoms). The numerical prescription follows simply: One calculates the real coordinates of the two atoms and uses the above equation to get $\phi_{i j}$. An important aspect of the Landau gauge is that the phase depends on the $x$ coordinate only through the difference of $x$ between the two atoms $i$ and $j$. This aspect is crucial for taking magnetic field into account in the leads: The leads are semi-infinite periodic systems made of layers. They are described by an intralayer Hamiltonian $H_{0}$ and an interlayer Hamiltonian $V$. Within the Landau gauge, we find that the matrices $H_{0}$ and $V$ of horizontal leads are layer independent, which allows the use of standard schemes to calculate their self-energies. However, general samples (such as the $\Psi$-shaped sample studied in this article) can have leads with an arbitrary angle $\theta$ with respect to the $y$ axis. For those leads, the corresponding gauge choice is

$$
\mathbf{A}(\mathbf{r})=-B\left(\mathbf{r} \cdot \mathbf{e}_{2}\right) \mathbf{e}_{1},
$$

with

$$
\mathbf{e}_{1}=\left(\begin{array}{c}
\cos \theta \\
\sin \theta
\end{array}\right), \quad \mathbf{e}_{2}=\left(\begin{array}{c}
-\sin \theta \\
\cos \theta
\end{array}\right)
$$

which leads to 


$$
\phi_{i j}=\phi_{i j}^{\mathrm{Lg}}+\Phi_{i}-\Phi_{j}
$$

where the potential

$$
\Phi_{i}=B(1-\cos 2 \theta) \frac{x_{i} y_{i}}{2}+B \sin 2 \theta \frac{x_{i}^{2}-y_{i}^{2}}{4}
$$

is a pure gauge potential allowing one to go from one choice of gauge to the other. A (possible) general prescription for an arbitrary system is now the following: One assigns a potential $\Phi_{i}=0$ to all sites except those belonging to a lead. For lead sites, one uses Eq. (A7) with the appropriate angle $\theta$. Then one calculates the phases $\phi_{i j}$ according to Eq. (A6).

All the prescriptions above are given in real space. It is of course possible to calculate analytically the equivalent prescriptions in terms of the integer coordinates on the Bravais sublattices, as both are in one-to-one correspondence. However, for numerical purposes, it is more convenient to calculate the real-space positions of the atoms numerically and then use Eqs. (A3), (A7), and (A6), in order to use a lattice-independent prescription.

[1] S. Das Sarma, S. Adam, E. H. Hwang, and E. Rossi, Electronic Transport in Two-Dimensional Graphene, Rev. Mod. Phys. 83, 407 (2011).

[2] M.Z. Hasan and C.L. Kane, Colloqium: Topological Insulators, Rev. Mod. Phys. 82, 3045 (2010).

[3] T. Ando, T. Nakanishi, and R. Saito, Berry's Phase and Absence of Backscattering in Carbon Nanotubes, J. Phys. Soc. Jpn. 67, 2857 (1998).

[4] A. F. Young and P. Kim, Quantum Interference and Klein Tunneling in Graphene Heterojunctions, Nature Phys. 5, 222 (2009).

[5] H. Suzuura and T. Ando, Crossover from Symplectic to Orthogonal Class in a Two-Dimensional Honeycomb Lattice, Phys. Rev. Lett. 89, 266603 (2002).

[6] F. V. Tikhonenko, A. A. Kozikov, A. K. Savchenko, and R. V. Gorbachev, Transition between Electron Localization and Antilocalization in Graphene, Phys. Rev. Lett. 103, 226801 (2009).

[7] K.S. Novoselov, A. K. Geim, S. V. Morozov, D. Jiang, M. I. Katsnelson, I. V. Grigorieva, S. V. Dubonos, and A. A. Firsov, Two-Dimensional Gas of Massless Dirac Fermions in Graphene, Nature (London) 438, 197 (2005).

[8] Y. Zhang, Y.-W. Tan, H.L. Stormer, and P. Kim, Experimental Observation of the Quantum Hall Effect and Berry's Phase in Graphene, Nature (London) 438, 201 (2005).

[9] M. O. Goerbig, Electronic Properties of Graphene in a Strong Magnetic Field, Rev. Mod. Phys. 83, 1193 (2011).

[10] C. L. Kane and E. J. Mele, Quantum Spin Hall Effect in Graphene, Phys. Rev. Lett. 95, 226801 (2005).

[11] C. L. Kane and E. J. Mele, $\mathbb{Z}_{2}$ Topological Order and the Quantum Spin Hall Effect, Phys. Rev. Lett. 95, 146802 (2005).
[12] B. A. Bernevig, T. L. Hughes, and S.-C. Zhang, Quantum Spin Hall Effect and Topological Phase Transition in HgTe Quantum Wells, Science 314, 1757 (2006).

[13] J.E. Moore and L. Balents, Topological Invariants of Time-Reversal-Invariant Band Structures, Phys. Rev. B 75, 121306 (2007).

[14] R. Roy, Topological Phases and the Quantum Spin Hall Effect in Three Dimensions, Phys. Rev. B 79, 195322 (2009).

[15] M. König, S. Wiedmann, C. Brüne, A. Roth, H. Buhmann, L. W. Molenkamp, X.-L. Qi, and S.-C. Zhang, Quantum Spin Hall Insulator State in HgTe Quantum Wells, Science 318, 766 (2007).

[16] C. Weeks, J. Hu, J. Alicea, M. Franz, and R. Wu, Engineering a Robust Quantum Spin Hall State in Graphene via Adatom Deposition, Phys. Rev. X 1, 021001 (2011).

[17] A. K. Geim, Graphene: Status and Prospects, Science 324, 1530 (2009).

[18] O. Shevtsov, P. Carmier, C. Groth, X. Waintal, and D. Carpentier, Tunable Thermopower in a Graphene-Based Topological Insulator, Phys. Rev. B 85, 245441 (2012).

[19] J. R. Williams and C.M. Marcus, Snake States along Graphene p-n Junctions, Phys. Rev. Lett. 107, 046602 (2011).

[20] G. Tkachov and E. M. Hankiewicz, Ballistic Quantum Spin Hall State and Enhanced Edge Backscattering in Strong Magnetic Fields, Phys. Rev. Lett. 104, 166803 (2010).

[21] Y. Yang, Z. Xu, L. Sheng, B. Wang, D. Y. Xing, and D. N. Sheng, Time-Reversal-Symmetry-Broken Quantum Spin Hall Effect, Phys. Rev. Lett. 107, 066602 (2011).

[22] A. De Martino, A. Hütten, and R. Egger, Landau Levels, Edge States, and Strained Magnetic Waveguides in Graphene Monolayers with Enhanced Spin-Orbit Interaction, Phys. Rev. B 84, 155420 (2011).

[23] N. Goldman, W. Beugeling, and C. Morais Smith, Topological Phase Transitions between Chiral and Helical Spin Textures in a Lattice with Spin-Orbit Coupling and Magnetic Field, Europhys. Lett. 97, 23003 (2012).

[24] D. A. Abanin, P. A. Lee, and L. S. Levitov, Spin-Filtered Edge States and Quantum Hall Effect in Graphene, Phys. Rev. Lett. 96, 176803 (2006).

[25] It is so weak that it appears to be superseded by manybody effects in strong magnetic fields [26,27]. If Zeeman splitting $\epsilon_{Z}$ were artificially enhanced, it would compete with SO coupling and lead to a phase transition when $\epsilon_{Z}=\Delta_{\text {so }}$, characterized by a reversal of the direction of spin current on a given edge [22].

[26] Y. Zhang, Z. Jiang, J. P. Small, M. S. Purewal, Y.-W. Tan, M. Fazlollahi, J. D. Chudow, J. A. Jaszczak, H. L. Stormer, and P. Kim, Landau-Level Splitting in Graphene in High Magnetic Fields, Phys. Rev. Lett. 96, 136806 (2006).

[27] Z. Jiang, Y. Zhang, H. L. Stormer, and P. Kim, Quantum Hall States near the Charge-Neutral Dirac Point in Graphene, Phys. Rev. Lett. 99, 106802 (2007).

[28] K. Kazymyrenko and X. Waintal, Knitting Algorithm for Calculating Green Functions in Quantum Systems, Phys. Rev. B 77, 115119 (2008). 
[29] D. J. Thouless, M. Kohmoto, M.P. Nightingale, and M. den Nijs, Quantized Hall Conductance in a TwoDimensional Periodic Potential, Phys. Rev. Lett. 49, 405 (1982).

[30] H. Watanabe, Y. Hatsugai, and H. Aoki, Manipulation of the Dirac Cones and the Anomaly in the Graphene Related Quantum Hall Effect, J. Phys. Conf. Ser. 334, 012044 (2011).

[31] D. N. Sheng, Z. Y. Weng, L. Sheng, and F. D. M. Haldane, Quantum Spin-Hall Effect and Topologically Invariant Chern Numbers, Phys. Rev. Lett. 97, 036808 (2006).

[32] This effect will be all the more potent for zigzagterminated ribbons, as they feature energy-dependent QSH edge states, whose width is expected to strongly increase when the energy approaches the SO gap [33].

[33] G. Metalidis and E. Prada, Transport through Quantum Spin Hall Insulator/Metal Junctions in Graphene Ribbons, arXiv: $1012.4345 v 2$.

[34] J. R. Williams, L. DiCarlo, and C.M. Marcus, Quantum Hall Effect in a Graphene p-n Junction, Science 317, 638 (2007).

[35] B. Ozyilmaz, P. Jarillo-Herrero, D. Efetov, D. A. Abanin, L.S. Levitov, and P. Kim, Electronic Transport and Quantum Hall Effect in Bipolar Graphene $p-n-p$ Junction, Phys. Rev. Lett. 99, 166804 (2007).

[36] D. A. Abanin and L.S. Levitov, Quantized Transport in Graphene p-n Junctions in a Magnetic Field, Science 317, 641 (2007).

[37] J. Tworzydlo, I. Snyman, A. R. Akhmerov, and C. W. J. Beenakker, Valley-Isospin Dependence of the Quantum
Hall Effect in a Graphene p-n Junction, Phys. Rev. B 76, 035411 (2007).

[38] A. R. Akhmerov, J. H. Bardarson, A. Rycerz, and C. W. J. Beenakker, Theory of the Valley-Valve Effect in Graphene Nanoribbons, Phys. Rev. B 77, 205416 (2008).

[39] P. Carmier, C. Lewenkopf, and D. Ullmo, Semiclassical Magnetotransport in Graphene n-p Junctions, Phys. Rev. B 84, 195428 (2011).

[40] Far from the Dirac point, however, the adatoms which induce the QSH phase in graphene [16] may give rise to parasitic [10] Rashba-type SO couplings.

[41] Note that a similar switching mechanism (without the spin polarization) could also be realized using a graphene quantum Hall $n$ - $p$ junction, provided the charge-density fluctuations close to the Dirac point can be reduced.

[42] K. S. Novoselov, E. McCann, S. V. Morozov, V. I. Fal'ko, M. I. Katsnelson, U. Zeitler, D. Jiang, F. Schedin, and A. K. Geim, Unconventional Quantum Hall Effect and Berry's Phase of $2 \pi$ in Bilayer Graphene, Nature Phys. 2, 177 (2006).

[43] E. McCann and V.I. Fal'ko, Landau Level Degeneracy and Quantum Hall Effect in a Graphite Bilayer, Phys. Rev. Lett. 96, 086805 (2006).

[44] Z. Qiao, W.-K. Tse, H. Jiang, Y. Yao, and Q. Niu, TwoDimensional Topological Insulator State and Topological Phase Transition in Bilayer Graphene, Phys. Rev. Lett. 107, 256801 (2011).

[45] H. U. Baranger and A.D. Stone, Electrical LinearResponse Theory in an Arbitrary Magnetic Field: A New Fermi-Surface Formation, Phys. Rev. B 40, 8169 (1989). 